\title{
Eddy Current Testing of the Lightning Strike Protection Layer in Aerospace Composite Structures
}

\author{
Bo FENG ${ }^{\text {a, } 1}$, Dario J. PASADAS ${ }^{\text {a }}$, Artur L. RIBEIRO ${ }^{\mathrm{a}}$ and Helena G. RAMOS ${ }^{\mathrm{a}}$ \\ a Instituto de Telecomunicações, Instituto Superior Técnico, Universidade de Lisboa, \\ Lisbon, Portugal
}

\begin{abstract}
The lightning strike protection layer, which is a mesh of metal stripes, is adhered to composite materials to dissipate the huge current induced during lightning strike. This paper presents an eddy current imaging method to inspect defects in the lightning strike protection layer. A tuning method was applied to tune the resonant frequency of excitation and sensing coils and enhance the testing results. Two parameters, namely the amplitude of induced voltage in the sensing coil and the amplitude of the voltage across a sampling resistor in the excitation circuit, were used to image the defects. The results show that the image formed by sensing coil voltage is less noisy and more accurate.
\end{abstract}

Keywords. Eddy current testing, composite, lightning strike protection layer

\section{Introduction}

Metals, especially aluminum and titanium, were extensively used to construct aerospace structures decades ago. Since 1990s, carbon fiber reinforced polymer (CFRP) composites have been increasingly used due to its high strength-to-density ratio and resistance to corrosion. Nowadays, composites can be the major part of the aircraft. For example, the Airbus A350 XWB is made of more than 50\% composites while the Airbus NH90 comprises $90 \%$ composites [1]. One of the main drawbacks of changing the aerospace structure from electrical conductive metal to semi-conducting composite is the vulnerability to lightning strike damage [2]. Lightning strikes, with current up to $200 \mathrm{kA}$, could cause fiber breakage, resin deterioration and delamination in composites. In order to protect the composite structure, a lightning strike protection layer, in the form of metal mesh, is commonly incorporated in the composite to dissipate currents [3].

The lightning strike protection layer could be damaged by huge current or corrosion and lose the ability to protect the composites. In order to guarantee its functionality, its integrity must be inspected. The purpose of this paper is to present an effective method for inspecting the breakages in the lightning strike protection layer and tries to improve the testing results.

${ }^{1}$ Corresponding Author, Bo FENG, Torre Norte Piso 11, Av. Rovisco Pais, 1, 1049-001 Lisboa, Portugal; E-mail: bofeng@tecnico.ulisboa.pt. 
Eddy current testing (ECT) is an efficient technique for detecting defects. In ECT, a time-varying voltage is send to a coil to generate time-varying magnetic field. When a conductive specimen is in the proximity of the coil, eddy currents are generated in the specimen and produce a secondary magnetic field. ECT has been widely used for inspecting metals due to their high conductivity [4-6]. With the emerging of new materials in industry in recent years, researchers have tried to apply ECT to inspect low-conductive composite materials [7-9]. The lightning strike protection layer is a metal mesh incorporated into composite base, therefore, ECT can be a possible method to detect the breakages in the lightning strike protection layer. However, further analysis and experiments should be done to study the effectiveness of ECT on this new structure.

\section{Materials and Methods}

\subsection{Samples to simulate lightning strike protection layer}

The lightning strike protection layer is a mesh of metals incorporated into the composite materials. Usually, the lightning strike protection layer is adhered onto the surface of the composite. There are also different types with the lightning strike protection layer located below several composite layers. It is well known that for the eddy current testing method, defects in the surface are easier to be detected than defects in the sub-surface. Therefore, a sample with lightning strike protection layer located inside the composite was made. The lay-up of the sample is shown in Figure 1. It consists five layers of CFRP material, each layer is with thickness of $0.8 \mathrm{~mm}$. Copper tapes, with thickness of $100 \mu \mathrm{m}$ and width of $5 \mathrm{~mm}$, were adhered onto the surface of the third layer to simulate a section of the metal mesh.

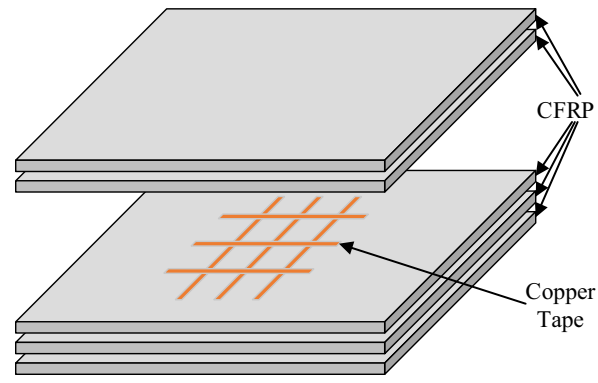

Figure 1. Lay-up of the sample.

When dissipating huge current during lightning strike, the metal mesh can be damaged due to the high temperatures attained because of Joule effect, and breakages can show up. In order to simulate the defects, the sample to be inspected was made incorporating three artificial breakages. A photo of the metal mesh described above and the defects is shown in Figure 2. The breakages were made with different width to test the resolution of the eddy current imaging system. 


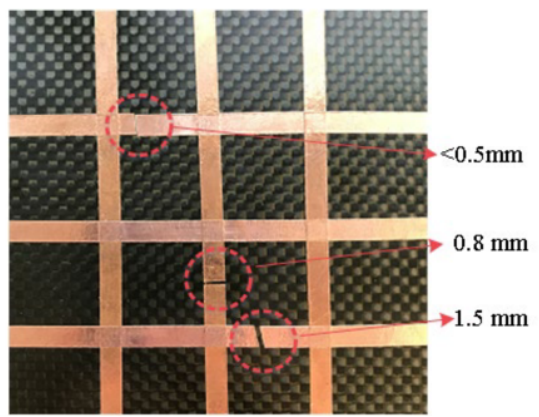

Figure 2. Artificial breakages in the sample.

\subsection{Eddy current testing probe and tuning technique}

The eddy current testing probe consists of an excitation coil outside and a coaxial sensing coil inside. The number of turns and dimensions of the excitation and sensing coils are listed in Table 1. As shown in Figure 3, the probe during inspection was placed above the composite sample with a lift-off of $1 \mathrm{~mm}$ and is fixed to a support of a two-axis positioning system.

Table 1. Specifications of excitation and sensing coils

\begin{tabular}{ccc}
\hline & Excitation coil & Sensing coil \\
\hline Number of turns & 391 & 50 \\
Height & $8 \mathrm{~mm}$ & $4 \mathrm{~mm}$ \\
Outer diameter & $23 \mathrm{~mm}$ & $4.5 \mathrm{~mm}$ \\
Inner diameter & $10 \mathrm{~mm}$ & $2 \mathrm{~mm}$ \\
\hline
\end{tabular}

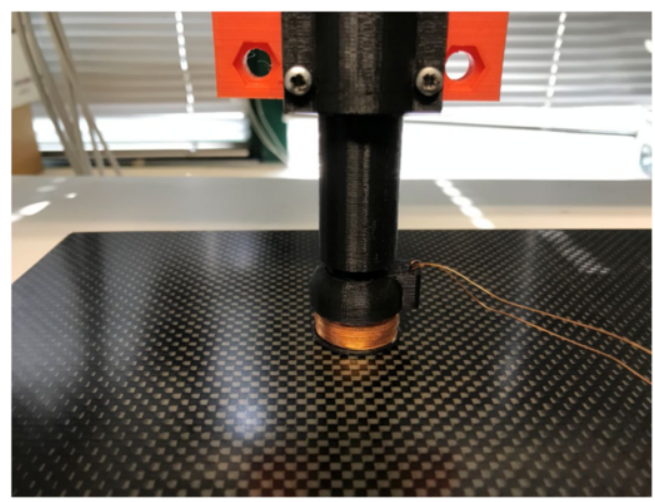

Figure 3. The eddy current testing probe.

In order to increase sensitivity, the excitation and the sensing circuits were tuned to the circuit resonant frequency. The resonant frequency of a LC circuit is given by:

$$
f=\frac{1}{2 \pi \sqrt{L C}}
$$

To tune the resonant frequency to the same value of $150 \mathrm{kHz}$, the inductances of the two coils were measured when they were in air. The tuning circuits are shown in Figure 4. A capacitor of $3.9 \mathrm{pF}$ was connected to the excitation coil in series to reduce the total impedance of the circuit and maximize the excitation current. A resistor of 
$10 \Omega$ was also added to the excitation circuit in series as a sampling resistor to monitor the excitation current. In the sensing circuit, a capacitor of $1.5 \mathrm{nF}$ was added to the sensing coil.

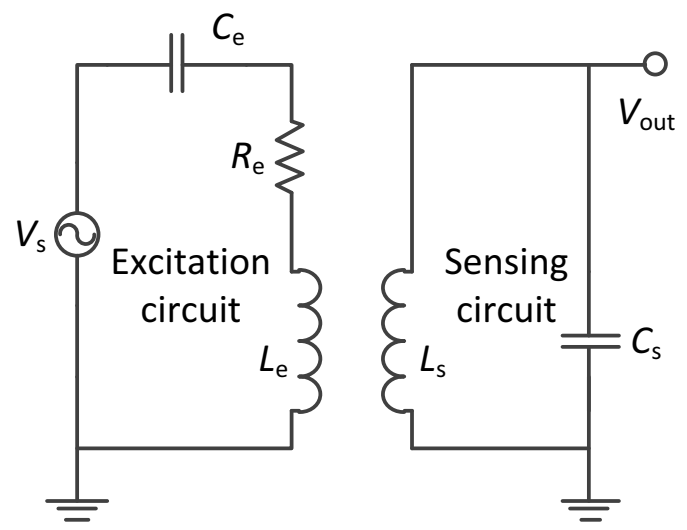

Figure 4. The tuning circuits for excitation and sensing coils.

\subsection{Measurement system}

A voltage with frequency of $150 \mathrm{kHz}$ and peak-to-peak amplitude of $1.8 \mathrm{~V}$ generated by an arbitrary function generator (Tektronix AFG 3102) was amplified by a power amplifier and applied to the excitation circuit. The ECT probe was driven by a two-axis positioning system to make a scan above the sample in an area of $81 \mathrm{~mm} \times 81 \mathrm{~mm}$ with steps of $1 \mathrm{~mm}$. At each location, two quantities were acquired by an oscilloscope (Tektronix TDS5032B Digital Oscilloscope) and were send to a computer. One quantity is the peak-to-peak amplitude of the voltage across the sampling resistor in excitation circuit, which reflects the change of impedance of the excitation coil caused by the defect. The other parameter is the peak-to-peak amplitude of the sensing coil voltage, which reflects the change of secondary magnetic field.

\section{Results}

After the scanning, a matrix with $81 \times 81$ elements was obtained in each channel of the oscilloscope. Each matrix directly shows the image of the defects. However, eddy current testing signals are prone to be influenced by lift-off changes. To reduce the influence of lift-off on the scanning results, a baseline matrix was built. In the matrix of the output voltage of the sensing coil, the value obtained at the starting point was subtracted from the values at the other three corners of the scanning region. The differences were used to form a baseline matrix of $81 \times 81$ points by interpolating linearly from the corners. The final result, which is shown in Figure 5, was obtained by subtracting the baseline matrix from the original matrix. From the ECT imaging results presented in Figure 5, it can be seen that the locations and dimensions of the copper tapes were accurately imaged, and at the junctions of two tapes, the ECT signals have larger perturbations. Furthermore, all the three breakages were successfully detected, as pointed out by the white circles. The perturbation increases with the width of the breakage. Another important detail to be noticed is the magnitude of the output voltage 
is $70 \mathrm{~V}$, even without amplification. The resonant technique effectively increased the output voltage.

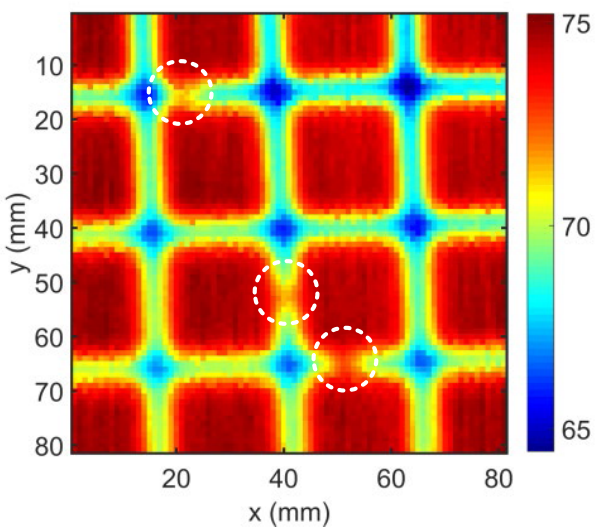

Figure 5. Output voltage of sensing coil.

The other quantity that can be used to image the defects is the voltage across the sampling resistor. The image was also adjusted to the change of lift-off by subtracting a baseline matrix. The result is shown in Figure 6. Even with baseline subtracting, the result is still noisy and severely influenced by the lift-off change. So, the defects are not able to be seen.

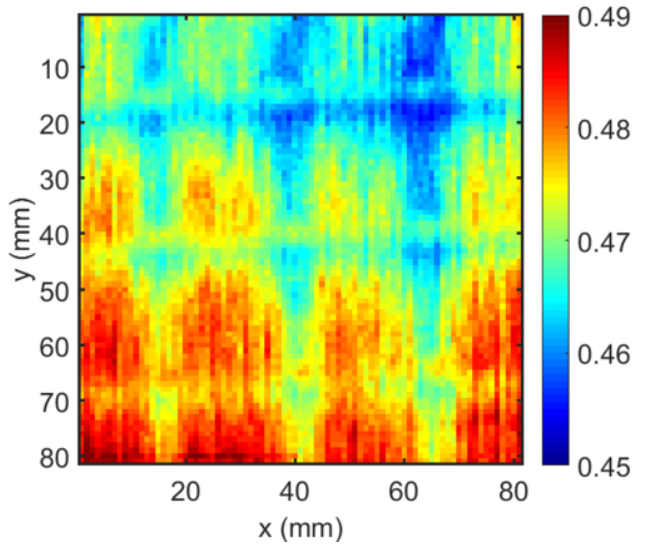

Figure 6. Voltage across sampling resistor.

\section{Conclusions}

This paper proposes an effective method for the inspection of breakages in the lightning strike protection layer in aerospace composite structures. An eddy current testing system with coaxial coils was used for the inspection. Both excitation and sensing circuits were tuned to the resonant frequency. This technique effectively increased the output voltage in the sensing coil. By comparing the results obtained using the output voltage at the sensing coil and the current that runs in the excitation current, it is concluded that the output voltage in the sensing coil is more suitable for imaging the defects. With the output voltage in the sensing coil, all the breakages in the sample were successfully detected even the smallest with a width of less than $0.5 \mathrm{~mm}$. 


\section{Acknowledgments}

This work was developed at Instituto de Telecomunicações and it was supported by the Portuguese Science and Technology Foundation (FCT) under projects UID/EEA/50008/2019 and PTDC/EEI/EEE/30723/2017. The supports are gratefully acknowledged

\section{References}

[1] https:/www.airbus.com/newsroom/news/en/2017/02/Material-evolution.html.

[2] T. M. Dhanya, C. S.Yerramalli, Lightning strike effect on carbon fiber reinforced composites - effect of copper mesh protection, Materials Today Communications 16 (2018), 124-134.

[3] M. Gagné, D. Therriault, Lightning strike protection of composites, Progress in Aerospace Sciences 64 (2014), 1-16.

[4] D. Pasadas, A. L. Ribeiro, T. Rocha, H. Ramos, 2D surface defect images applying Tikhonov regularized inversion and ECT, NDT \& E International 80 (2016), 48 - 57.

[5] F. Jiang, S. Liu, S. Xin, et al, Evaluation of circumferential cracks in metal tubes based on a magnetic field response model of eddy current testing, Insight 62 (2020), 91-97.

[6] Y. Yu, K, Gao, B. Liu, et al, Semi-analytical method for characterization slit defects in conducting metal by Eddy current nondestructive technique, Sensors and actuators a-physical 301 (2020), 111739.

[7] Z. Cai, D. Zou, C. Liu, Research on Eddy-Current Testing of Functional Polymer Composite Material, IEEE transactions on magnetics 54 (2018), 2501005.

[8] M.P. Degoeje, K. Wapenaar, Nondestructive inspection of carbon fiber-reinforced plastics using eddycurrent methods, Composites 23 (1119), 147-157.

[9] B. Feng, D.J. Pasadas, A.L. Ribeiro, H.G. Ramos, Locating and Imaging Impact Damage with an Integrated System of PZT and Eddy Current Probe, in Proc. Int. Instrum. Meas. Technol. Conf., Auckland, New Zealand, May 2019. 